\title{
Spatially mapping thermal transport in graphene by an opto- thermal method
}

\author{
Oliver Braun $\mathbb{D}^{1,2}$, Roman Furrer $\mathbb{D}^{1}$, Pascal Butti ${ }^{1}$, Kishan Thodkar ${ }^{1,4}$, Ivan Shorubalko ${ }^{1}$, Ilaria Zardo $\mathbb{D}^{2}$, Michel Calame $\mathbb{D}^{1,2,3}$ and \\ Mickael L. Perrin (iD ${ }^{1 凶}$
}

Mapping the thermal transport properties of materials at the nanoscale is of critical importance for optimizing heat conduction in nanoscale devices. Several methods to determine the thermal conductivity of materials have been developed, most of them yielding an average value across the sample, thereby disregarding the role of local variations. Here, we present a method for the spatially resolved assessment of the thermal conductivity of suspended graphene by using a combination of confocal Raman thermometry and a finite-element calculations-based fitting procedure. We demonstrate the working principle of our method by extracting the two-dimensional thermal conductivity map of one pristine suspended single-layer graphene sheet and one irradiated using helium ions. Our method paves the way for spatially resolving the thermal conductivity of other types of layered materials. This is particularly relevant for the design and engineering of nanoscale thermal circuits (e.g. thermal diodes).

npj 2D Materials and Applications (2022)6:6; https://doi.org/10.1038/s41699-021-00277-2

\section{INTRODUCTION}

Thermal properties of materials are of crucial importance for optimizing heat management in nanoscale devices, with the thermal conductivity as key material property ${ }^{1,2}$. The thermal conductivity is typically determined by monitoring the sample temperature and/or heat flow in response to a local heat source, in combination with an analytical expression or a numerical model. For instance, for bulk materials, the well-known $3 \omega$ technique $^{3,4}$ is used, while for nanoscale materials, methods such as the thermal bridge method ${ }^{5-7}$ and micro-Raman spectroscopy ${ }^{8-11}$ provide the thermal conductivity of the material.

Of particular interest are the thermal properties of layered materials. Due to their broad range of conductivity values and their atomically thin nature, such materials are highly relevant for heat management at the nanoscale ${ }^{12,13}$. One of the most appealing materials is graphene, with extraordinarily high thermal conductivity values. However, extracting the thermal properties of $2 \mathrm{D}$ materials is challenging, in particular when suspended to reduce the influence of the substrate. For example, time-domain thermoreflectance cannot be applied to $2 \mathrm{D}$ materials as the material is too thin ${ }^{14}$. Scanning thermal probe microscopy, on the other hand, despite possessing nanometer resolution, is highly delicate to perform on suspended 2D materials. Moreover, both techniques rely on on-chip heaters for channeling heat into the system. Raman spectroscopy can overcome these difficulties, as it can utilize the excitation laser to locally heat the device, while at the same time measuring the local temperature. Moreover, Raman spectroscopy can be conveniently performed on suspended graphene films for eliminating the influence of the substrate. Using Raman spectroscopy, Balandin and Ghosh et al. ${ }^{15,16}$ determined the thermal conductivity of suspended graphene to be as high as $\sim 5000 \mathrm{Wm}^{-1} \mathrm{~K}^{-1}$ at room temperature. Their optothermal method, measuring the shift of the Raman G-band upon laser irradiation for estimating the local temperature, has been extensively used in literature since. Alternatives based on the intensity ratio of Stokes to anti-Stokes Raman scattering ${ }^{17}$ or the Raman 2D-band ${ }^{18}$ have also been reported.

Using this opto-thermal method, the influence of the quality and structure of the graphene, as well as the environment have been extensively investigated. For instance, Cai et al..$^{19}$ reported values for $K$ exceeding $\sim 2500 \mathrm{Wm}^{-1} \mathrm{~K}^{-1}$ for suspended graphene grown by chemical vapor deposition (CVD), and Chen et al. ${ }^{20}$ studied the influence of environment on thermal conductivity of graphene. Isotopically pure ${ }^{12} \mathrm{C}\left(0.01 \%{ }^{13} \mathrm{C}\right)$ graphene has been shown to exhibit $K=4000 \mathrm{Wm}^{-1} \mathrm{~K}^{-1}$, a factor of two higher than $K$ in graphene composed of a 1:1 mixture of ${ }^{12} \mathrm{C}$ and ${ }^{13} \mathrm{C}^{21}$. Also, the influence of CVD-graphene's polycrystallinity on the thermal conductivity was studied by Lee et al. and Ma et al., revealing that smaller grain sizes drastically reduce $\kappa$ due to grain boundary scattering ${ }^{22,23}$. Along similar lines, wrinkles ${ }^{24}$, oxygen-plasma induced defects ${ }^{25}$, and electron beam irradiation ${ }^{26}$ have shown to reduce the thermal conductivity.

In all the above-mentioned studies, the one-dimensional heat equation is used to fit the experimental temperature and extract the thermal conductivity. However, such approaches yield an average value of thermal conductivity value, not a spatially resolved map. This implied that local variations caused by defects, folds, contaminants, etc. are neglected.

For going beyond the average material property value, approaches mapping the temperature distribution in the sample are necessary, combined with multi-dimensional analytical or numerical model. A range of techniques has been developed for nanoscale thermometry, such as time-domain thermoreflectance ${ }^{27}$, Raman spectroscopy ${ }^{15,16}$, scanning thermal probe microscopy ${ }^{28-30}$, polymer imprint thermal mapping ${ }^{31}$ and electron energy loss spectroscopy ${ }^{32}$, providing means to locally map the sample temperature.

\footnotetext{
${ }^{1}$ Empa, Swiss Federal Laboratories for Materials Science and Technology, Transport at Nanoscale Interfaces Laboratory, Überlandstrasse 129 , CH-8600 Dübendorf, Switzerland.

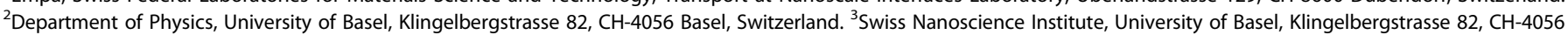
Basel, Switzerland. ${ }^{4}$ Present address: Department of Mechanical and Process Engineering, ETH Zurich, Tannenstrasse 3, CH-8092 Zurich, Switzerland.

email: Mickael.Perrin@Empa.ch
} 

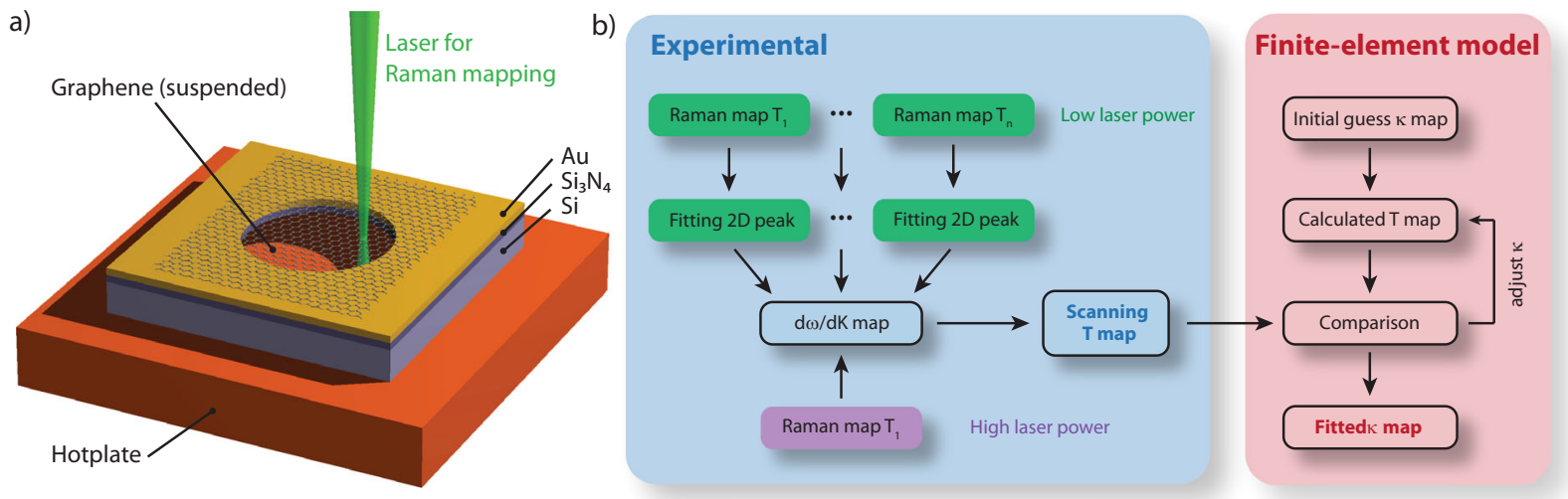

Fig. 1 Experimental and finite-element method description. a Schematic drawing of the suspended graphene membrane. b Experimental workflow to obtain a temperature map upon laser illumination and computational workflow to fit the corresponding thermal conductivity map.

Here, we introduce an opto-thermal method that allows for two-dimensional mapping of thermal conductivity of suspended graphene membranes. The presented method relies on a combination of scanning $\mu$-Raman spectroscopy finite-element method (FEM) calculations. The workflow for our approach is presented in Fig. 1. In the first experimental stage, a series of twodimensional Raman spectroscopy maps are used to construct a temperature map of the membrane upon illumination. More specifically, Raman maps are recorded at low laser power for various hotplate temperatures. This series of maps is used to construct a calibration map of the Raman peak shifts with temperature. Then, another Raman map is recorded at high laserpower, which, combined with the calibration map, is used to construct a temperature map of the membrane upon laser heating.

The constructed experimental temperature map is then used as an input for the FEM-based fit procedure. For a given initial guess of the thermal conductivity, the lattice temperature upon laser illumination is calculated. The thermal conductivity is then iteratively adjusted, until the computed temperature map matches the experimental one. We apply this fit procedure to extract the thermal conductivity of a pristine graphene membrane that is suspended over a silicon nitride frame. Finally, we demonstrate that the thermal conductivity of the graphene membrane can be tuned in a controlled way by the introduction of helium-ion ( $\mathrm{He}^{+}$-ion) induced defects in the membrane.

\section{RESULTS}

\section{Experimental temperature maps}

The CVD-graphene membranes are prepared as described in the methods section. We applied Raman spectroscopy to obtain the lattice temperature of the suspended graphene membranes (for details see Methods and Supplementary Notes 1 and 3). Here, we focus on the 2D-band due to its high sensitivity to temperature changes ${ }^{20,22}$ of around $-0.07 \mathrm{~cm}^{-1} \mathrm{~K}^{-1}$. Alternatively, one can also rely on the G-peak due to its high linearity in peak shift versus temperature. ${ }^{16,19,33}$.

Figure $2 \mathrm{a}$ presents maps of the Lorentzian-fitted 2D-peaks acquired at temperatures $T_{1}-T_{7}$ ranging between 298 and $425 \mathrm{~K}$. The Raman spectra have been acquired at low laser power $(0.25 \mathrm{~mW})$ to limit any heating effects using a $532 \mathrm{~nm}$ excitation laser (for details see Methods and Supplementary Note 3). For each pixel, the peak shift with hot plate temperature $\left(d \omega_{2 \mathrm{D}} / d T\right)$ is fitted using a first-order polynomial, as shown in Fig. $2 \mathrm{~b}$. The inset presents a histogram of the slopes, showing a Gaussian distribution centered around $-0.07 \mathrm{~cm}^{-1} \mathrm{~K}^{-1}$. The spatial distribution of the peak shifts with temperature is displayed in Fig. $2 c$, showing substantial spatial variations. Once this calibration map is acquired, a Raman map of the graphene membrane is acquired at high laser power ( $4 \mathrm{~mW})$, as shown in Fig. $2 \mathrm{~d}$. The high laser power causes the graphene to locally heat, resulting in a shift in the Raman peak position. We assume steady state conditions for all data interpretations as the thermal time constant is short $(\tau<$ $300 \mathrm{~ns}$ ) in suspended graphene ${ }^{34}$.

By combining this high-power measurement with the $d \omega_{2 D} / d T$ map obtained at low laser power, a map of the average temperature within the laser spot is obtained for each laser position, as shown in Fig. $2 \mathrm{~b}$. We note that local variations in the temperature upon illumination on the order of $50-100 \mathrm{~K}$ are observed, highlighting the importance of spatially mapping the temperature and its superiority over other techniques that extract thermal conductivity from a single spot.

\section{FEM calculations}

FEM calculations are employed for the computation of the temperature map of the system upon laser illumination for a given spatial thermal conductivity distribution. This concept is illustrated in Fig. 3. As an input, a two-dimensional map of the thermal conductivity is provided, of which an example is shown Fig. 3a. Figure 3b presents the layout of the system (not to scale). A detailed description of the FEM calculation and the implementation thereof is provided in Supplementary Note 3. While scanning the laser across the membrane, the full temperature distribution is calculated for each laser spot position on the membrane (three examples are provided in Fig. $3 \mathrm{~b}$ ).

For each of these temperature distributions, the average temperature within the laser spot is calculated, from which all values are combined to obtain a two-dimensional map of the graphene temperature upon illumination. This inducedtemperature map is presented in Fig. $3 c$ and represents the same temperature map that is obtained experimentally upon illumination of the sample with high laser power.

To obtain the thermal conductivity map, an iterative minimization procedure is employed. More information can be found in Supplementary Note 2, in which we also validate the numerical method on a simulated system with a known thermal conductivity map. The starting point is an initial (typically uniform) guess of the thermal conductivity. In each iteration of the process, the corresponding induced temperature map is compared to the experimental temperature map, after which the thermal conductivity is adjusted pixel-wise according to the temperature difference. This process is repeated until convergence is reached. 

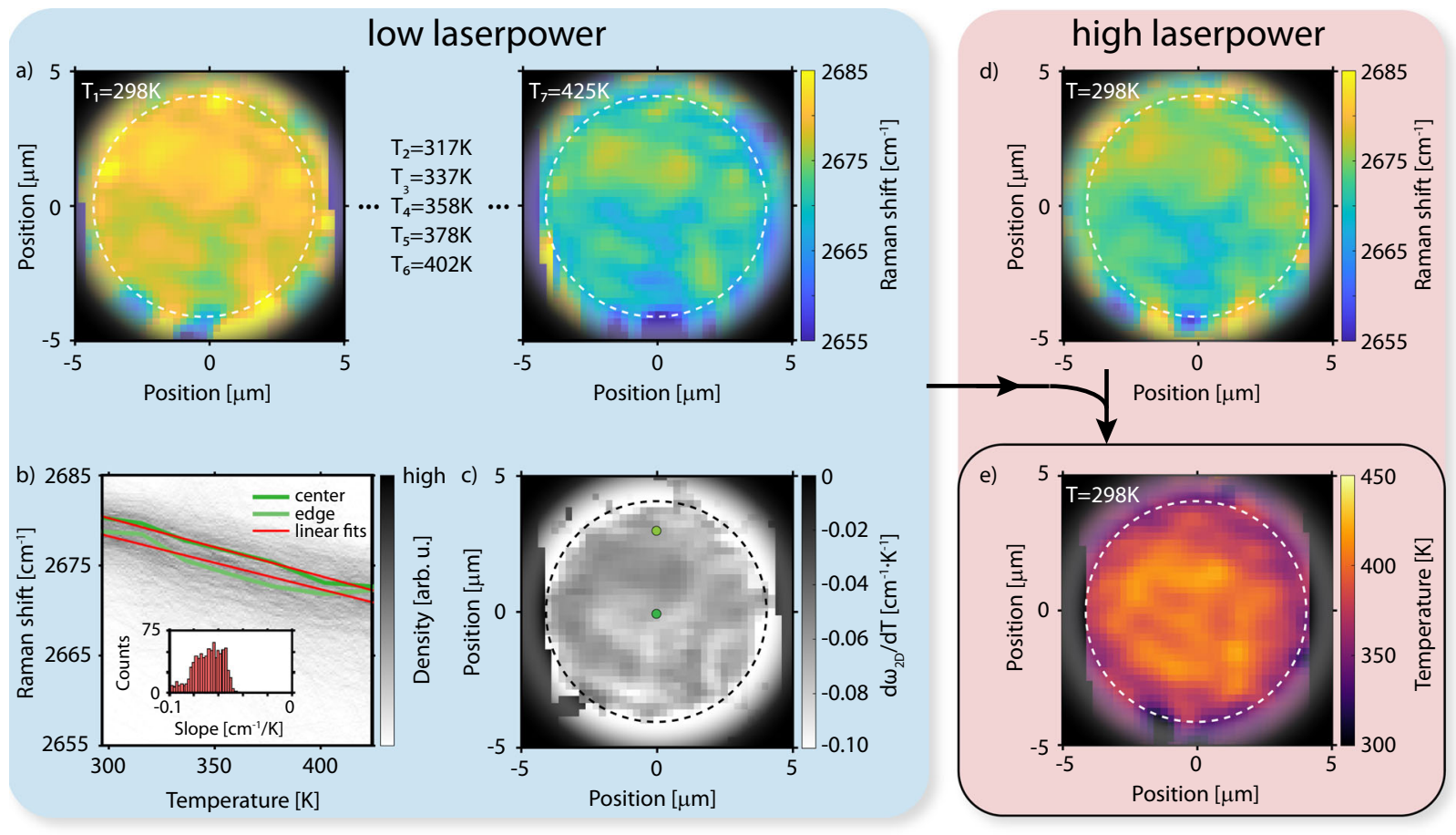

Fig. 2 Experimental determination of temperature map. Spatially resolved mapping of laser-induced temperature rise of graphene. a Raman 2D-peak position obtained with $P_{\text {laser }}=0.25 \mathrm{~mW}$ at different hot plate temperatures $\mathrm{T}_{1}$ and $\mathrm{T}_{7}$. The dashed circle is a guide to the eye for the support edge. b Density plot of the temperature evolution of the 2D-peak frequency. Two spatial points (center and edge, see dots in c are highlighted to represent the method. The inset shows a histogram of $d \omega_{2 \mathrm{D}} / d T$ of the complete membrane. c Spatial distribution of change in Raman shift per temperature change $d \omega_{2 \mathrm{D}} / d T$ obtained from linear fits to the data shown in b). d Raman 2D-peak position obtained with $P_{\text {laser }}=4 \mathrm{~mW}$ at $297 \mathrm{~K}$. e Temperature distribution obtained by combining the results from c, $\mathbf{d}$.

a)

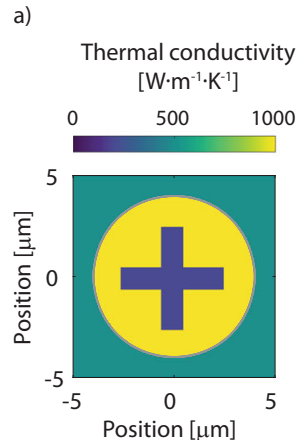

b) Supported

$$
\text { graphene }
$$

Suspended graphene

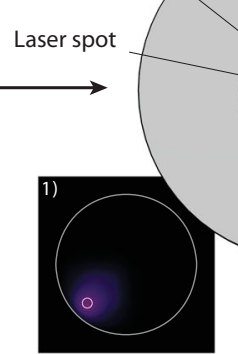

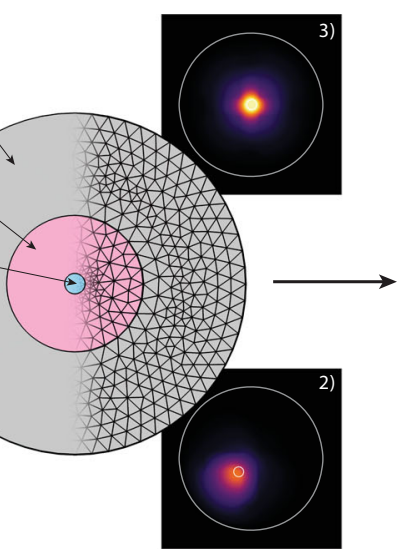

c)

$$
\begin{aligned}
& \text { Temperature upon } \\
& \text { illumination }[\mathrm{K}]
\end{aligned}
$$

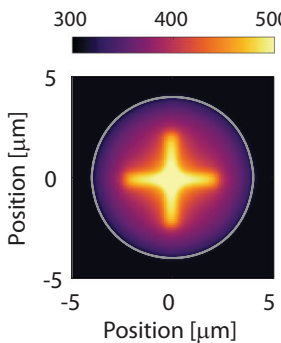

Fig. 3 Finite-element method description. a Input thermal conductivity map. $\mathbf{b}$ Schematic representation of the sample and the calculation mesh. Temperature profiles of the graphene membrane with the heating laser spot at three different positions (1-3). c Temperature profile upon laser illumination.

\section{Thermal conductivity map based on fitted experimental temperature map}

The induced temperature map obtained in Fig. 2 is used as input for the iterative procedure to obtain the thermal conductivity map. Here, as clarified in Supplementary Note 3, we use a uniform absorption of $2.7 \%$ for the suspended graphene and double that value (5.4\%) for the supported graphene. Moreover, all the employed model parameters are summarized in Supplementary Table 1. Importantly, in the model, a spot size of $370 \mathrm{~nm}$ is considered, based on a measurement shown in Supplementary Note 3. This value is larger than the diffraction-limit, based on the Abbe criterion $d=\lambda / 2 \mathrm{NA}$, where $d$ is the spotsize, $\lambda$ is the wavelength and NA is the numerical aperture $^{35}$. Moreover, the thermal conductivity of graphene on the supporting part, the thermal coupling to the substrate, as well as the convection parameter are taken from literature ${ }^{19}$. Finally, we note that it is challenging to model the transition from the suspended graphene to the supported graphene once the laser spot is in the vicinity of the edge due to (1) reflection from of the laser excitation at the edges of the support may lead to an increase in the deposited laser power, (2) quenching of the Raman scattered light on the substrate may lead to an overestimation of the local temperature as the $2 \mathrm{D}$ peak of the suspended graphene is more pronounced than that of the supported graphene. To circumvent this issue, the thermal conductivity of the first $0.5 \mu \mathrm{m}$ of the membranes away from the support are not fitted and kept at a fixed value. Finally, the thermal conductivity is fitted for 100 iterations, after which the absorption is 

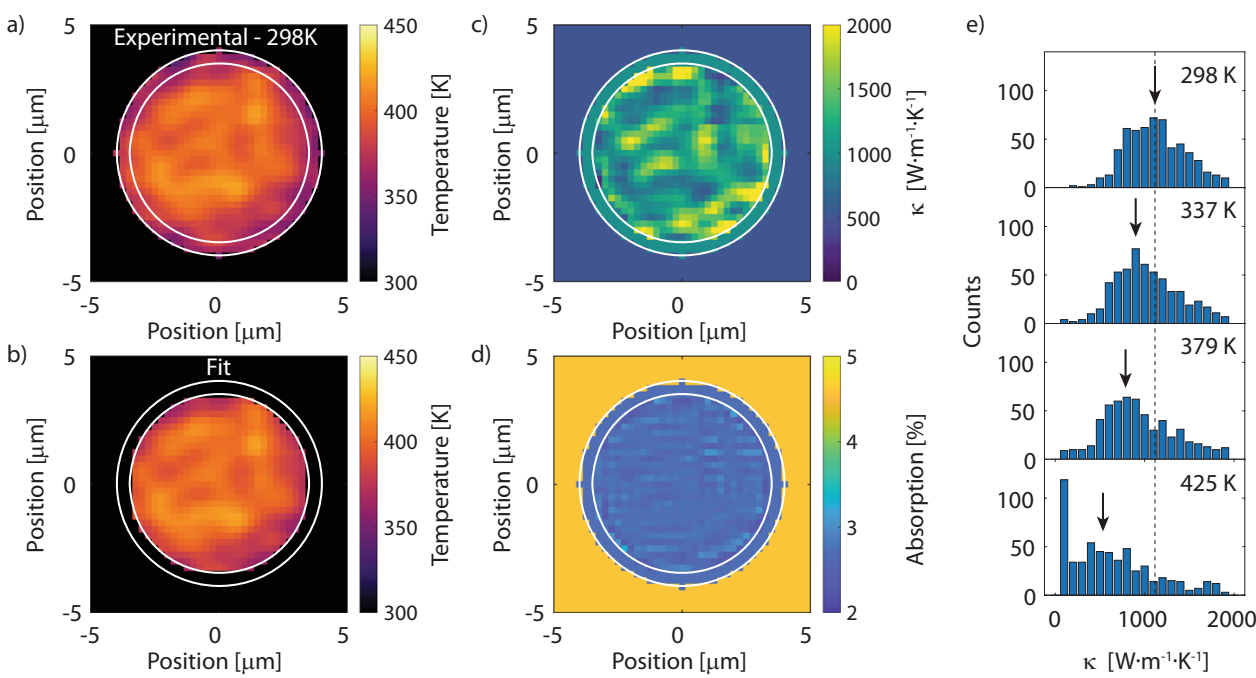

Fig. 4 Thermal conductivity map based on fitted experimental data. a Experimentally determined temperature map. b Fitted temperature map. c Fitted thermal conductivity map. d Converged absorption map. e Histogram of the thermal conductivity for various hot plate temperatures. Arrows indicate the mode of the smoothed distributions.

fitted for the same number of cycles. More details about this procedure can be found in Supplementary Note 2. For numerical stability reasons, we put a lower value on the thermal conductivity at $100 \mathrm{Wm}^{-1} \mathrm{~K}^{-1}$.

Figure $4 \mathrm{a}$ presents the experimental temperature map, as obtained in Fig. 2e, alongside the fitted temperature map in Fig. 4b. The two maps closely resemble each other. The corresponding thermal conductivity map is presented in Fig. 4c. We find that the local thermal conductivity values range from 500 to $2000 \mathrm{Wm}^{-1} \mathrm{~K}^{-1}$ with an average value of $1224 \pm 387 \mathrm{Wm}^{-1} \mathrm{~K}^{-1}$ highlighting the importance of spatially resolving the thermal conductivity. The average value is in agreement with previously reported values for CVD-graphene, where typical defects like polymer residues, grain boundaries, etc. are present ${ }^{36-39}$. In Fig. 4 e, we present a histogram of the fitted thermal conductivity map for increasing hot plate temperatures. The bar plots show that for increasing temperature a gradual decrease in thermal conductivity is observed. This behavior follows the trend observed by others ${ }^{20-22}$.

\section{Thermal conductivity of defect-engineered graphene}

As a final demonstration of the capability of the presented method, we study the thermal conductivity of a graphene membrane that is exposed to $\mathrm{He}^{+}$-ions using focused ion beam (FIB) lithography. As shown previously, $\mathrm{He}^{+}$-ions can be used to induce, in a controlled fashion, defects in suspended graphene membranes and other two-dimensional materials ${ }^{40,41}$.

Figure $5 a$ presents the exposure pattern as well as the used irradiation doses. The membrane is divided into four quadrants, with the $\mathrm{He}^{+}$-ion irradiation steadily increasing in the counterclockwise direction, starting in the lower left with no $\mathrm{He}^{+}$-ion dose. Manually selected representative Raman spectra of each quadrant are presented in Supplementary Note 4, exhibiting all the characteristic graphene peaks. The selection of the representative Raman spectra can also be done by an advanced clustering approach to avoid any human bias as described elsewhere ${ }^{42}$. Upon an increase of the $\mathrm{He}^{+}$-ion dose, the D-band intensity steadily increases. The intensity ratio of the $D$ and $D^{\prime}$ band I(D)/I(D') upon irradiation, is indicative of the type of defect. ${ }^{43}$. We extract this ratio by fitting the ratios $I(D) / I(G)$ versus $I\left(D^{\prime}\right) / I(G)$ for various $\mathrm{He}^{+}$-ion doses. We find an intensity ratio of $\sim 11.7$ for the defectengineered graphene. This value is comparable to the reported intensity ratio of $\sim 13$ for $\mathrm{sp}^{3}$ type of defects (see Supplementary Note 4). We employ the same procedure as presented in Fig. 2 for the extraction of the temperature. Figure $5 \mathrm{~b}$ presents the induced temperature map upon a $4 \mathrm{~mW}$ laser illumination. In this plot, the four quadrants are visible, with the lowest temperatures recorded in the (unexposed) lower left section of the membrane, and the highest one in the upper left (most exposed). This temperature map is used as input for the iterative FEM-based fitting procedure, resulting in the fitted thermal conductivity map in Fig. $5 \mathrm{c}$ and the corresponding fitted temperature map shown in Fig. 5d. Figure $5 \mathrm{e}$ presents a histogram of the thermal conductivity of each of the four quadrants. A steady decrease in average conductivity is observed, from $\sim 1200 \mathrm{Wm}^{-1} \mathrm{~K}^{-1}$ for the no $\mathrm{He}^{+}$-ion irradiation, to $\sim 300 \mathrm{Wm}^{-1} \mathrm{~K}^{-1}$ for the highest $\mathrm{He}^{+}$-ion dose. This decrease in thermal conductivity with increasing defect density is in agreement with previous reports ${ }^{25,26}$.

\section{DISCUSSION}

The FEM calculations employed here assume that the heat transport through the system is following Fourier's Law. This assumption implies that the phonon mean free path is much smaller than the membrane size. Indeed, ballistic phonon transport has been reported in several nanosystems at room temperature: In substrate-supported graphene the phonon mean free path is $\sim 100 \mathrm{~nm}^{44}$; for suspended graphene discs, the transition from ballistic to diffusive transport occurs at $\sim 775 \mathrm{~nm}^{45}$ while in ultra-thin nanowires phonon mean free paths of several micrometers have been observed ${ }^{46}$. Therefore, the resolution of the presented method is limited by the phonon mean free path as a spatial mapping of the thermal conductivity below this length scale would require a heat transport description based on the Boltzmann transport equation ${ }^{47-49}$. Given this boundary condition, the resolution of $250 \mathrm{~nm}$ used in this study is close to the ultimate resolution this FEM-based method allows.

A limitation of the presented method is the time consumption of the temperature calibration, reducing its use in highthroughput applications. As the laser power is low, acquiring the two-dimensional Raman map at each temperature requires several hours. This long acquisition time can also lead to a drift in the sample position during the measurement. To reduce this drift, clamping of the sample and a good thermalization of the sample with the environment is crucial. Furthermore, as changing the hot plate temperature leads to shifts of the sample position, the Raman maps acquired at various temperatures need to be aligned one versus the other. 

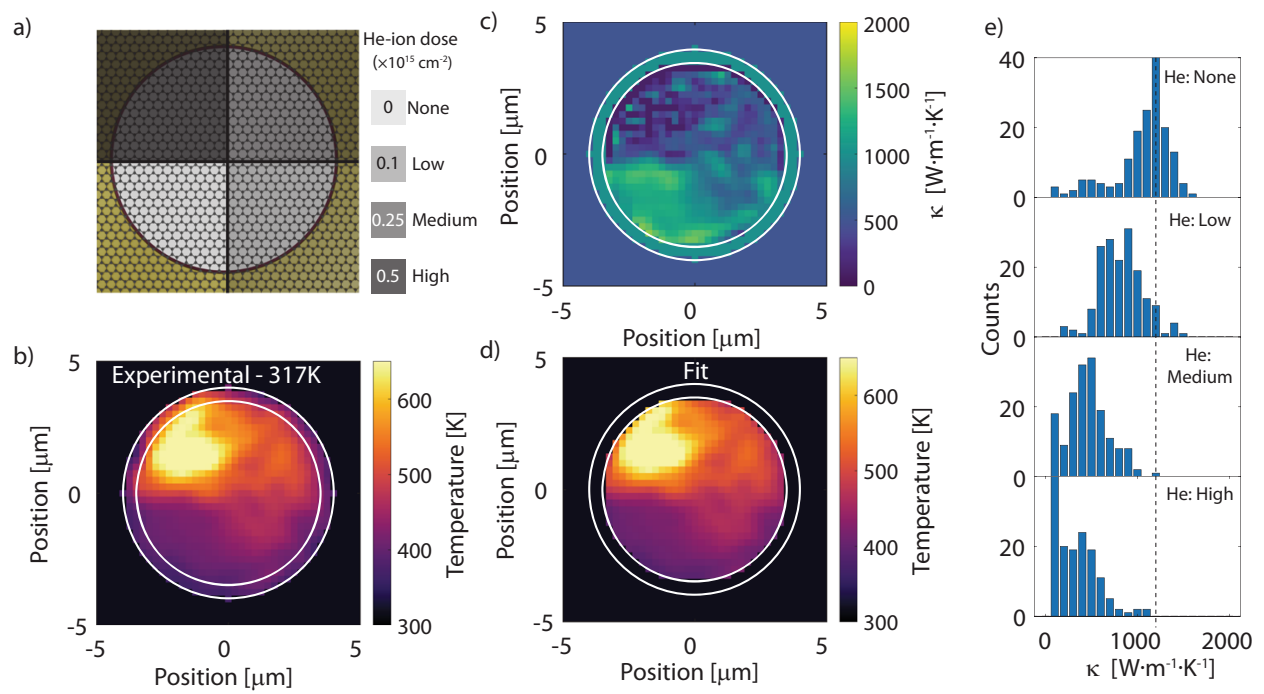

Fig. 5 Thermal conductivity of defect-engineered graphene. a Schematic image of a suspended graphene membrane. The areas where the membrane was exposed to $\mathrm{He}^{+}$-ions and their corresponding dose is indicated with different colors. $\mathbf{b}$ Experimentally observed temperature map. c Fitted thermal conductivity map. d Fitted temperature map. e Histogram of the thermal conductivity for various defect densities.

A second limitation is the fixed value for the absorption of $2.7 \%$ that is used for the first 100 cycles of the fitting procedure, after which the absorption is fitted as well. The accuracy of the model may be improved by experimentally determining the absorption at the various hot plate temperatures. Ideally, the absorption would be measured by simultaneously monitoring the transmitted, and reflected laser power while scanning across the sample. We stress that simultaneously measuring both components is crucial, as contaminations and residues on the membrane may scatter the laser light, leading to a reduction in the transmitted light, but not to an increase in absorption. However, such a measurement is challenging and technically unfeasible in our current setup.

Despite the previously mentioned limitations, our method is well suited for studying the thermal properties of two-dimensional materials, in particular for materials with an anisotropic thermal conductivity ${ }^{50-52}$. The method can also be extended to characterize van der Waals materials consisting of multiple layers. Furthermore, the method is extensible from two to three dimensions, allowing for modeling of more complex device geometries, including, for instance, stacks of 2D-materials, or the presence of contact electrodes of finite thickness. As such, it could be used for assessing the material quality after device integration. Also, as the individual two-dimensional materials in a stacked geometry each have a distinct Raman signature, it is possible to investigate the subsurface thermal properties of materials, such as, for instance, graphene embedded in a thin hexagonal boron nitride layer. Alternatively, when the material under study is on a substrate or thick enough, other means of determining the temperature map may be used, like time-domain thermoreflectance, for reduced measurement time and improve throughput.

We have introduced a method for spatially mapping the thermal conductivity of single-layer graphene using a combination of Raman spectroscopy and finite-element calculations at ultimate resolution. We anticipate that this method can be applied to other single- and few layer materials. We applied the method to obtain the thermal conductivity of a pristine and $\mathrm{He}^{+}$-ion patterned suspended single-layer graphene film. For the unpatterned film, large variations of the extracted thermal conductivity are observed and attributed to local irregularities such as contamination, defects, or folds. These findings highlight the importance of spatial mapping of the thermal conductivity, in contrast to measurement approaches that yield a thermal conductivity averaged across the entire sample. On the patterned membrane, we demonstrate controlled engineering of the thermal conductivity by $\mathrm{He}^{+}$-ion irradiation. As Raman spectroscopy is widely used in the two-dimensional materials community, our method is ideally suited for studying the thermal properties of other layered materials. Moreover, the working principle of the FEM method can easily be extended to more complex geometries or interfaces, in particular combined with alternative measurement techniques for providing a temperature map. Our method enables spatially resolving the thermal conductivity of atomically thin materials, a prerequisite for optimizing and engineering thermal stewardship in nanoscale devices.

\section{METHODS}

\section{Preparation of the SiN membrane}

Two types of $\mathrm{Si} / \mathrm{Si}_{3} \mathrm{~N}_{4}$-membranes were used. First, commercially available $\mathrm{Si} / \mathrm{Si}_{3} \mathrm{~N}_{4}$-membranes (Norcada Inc., NORCADA Low Stress SiNx Membrane NX5200D) were patterned with arrays of holes of various diameters using Gallium-FIB (FEI, Strata), see Supplementary Note 1. Second, silicon nitrite frames are fabricated using dry and wet etch processes as described elsewhere ${ }^{53}$. Further, a Ti/Au $(5 / 40 \mathrm{~nm})$ layer is deposited using an electron beam evaporator to ensure thermal anchoring.

\section{Synthesis and transfer of graphene}

The CVD-graphene is synthetized as described here and previously ${ }^{40,54,55}$. The single-layer graphene samples were synthesized using a Cu metal catalyst by CVD. A $25 \mu \mathrm{m}$ thick Cu-foil was cleaned with acetic acid for $20 \mathrm{~min}$ and rinsed with DI-water and ethanol. The Cu-foil was then heated up to $1000^{\circ} \mathrm{C}$ inside a quartz tube under $\mathrm{Ar}$ atmosphere for $3 \mathrm{~h}$, and the graphene was then grown with flowing gas mixtures of $\mathrm{Ar}: \mathrm{H}_{2}: \mathrm{CH}_{4}=$ 200:20:0.1 (sccm) for $60 \mathrm{~min}$. After synthesizing the graphene, the polymethyl-methacrylate (PMMA) was coated on the graphene at 3000 RPM for $30 \mathrm{~s}$. Using reactive ion etching $\left(\mathrm{Ar} / \mathrm{O}_{2}\right.$-Plasma, $\left.60 \mathrm{~s}\right)$ the graphene on the back-side of the PMMA/graphene/metal catalyst was removed. The metal catalyst was then etched by floating on a $0.1 \mathrm{M}$ ammonium persulfate solution overnight. After rinsing the PMMA/graphene with DIwater, the PMMA/graphene was transferred onto the target substrate and baked at $110^{\circ} \mathrm{C}$ for $30 \mathrm{~min}$ with an intermediate step at $80^{\circ} \mathrm{C}$ for $10 \mathrm{~min}$, increasing the adhesion between the graphene and target substrate. The PMMA was removed with acetone, IPA followed by DI-water. For this study also graphene grown with a slightly different recipe as reported elsewhere was used. Besides graphene grown by us, also commercially available graphene (Easy Transfer, Graphenea and graphene grown and transferred 
by Applied Nanolayers) was used. A scanning electron micrograph is provided in Supplementary Fig. 1.

\section{Raman setup and spectra analysis}

Raman spectra were acquired with a confocal Raman microscope (WITec, Alpha $300 \mathrm{R})$ in backscattering geometry, equipped with a $100 \times(\mathrm{NA}=0.9)$ and a $50 \times(\mathrm{NA}=0.55$, long working distance) objective lenses. The backscattered light was coupled to a $300 \mathrm{~mm}$ lens-based spectrometer with gratings of $600 \mathrm{~g} \mathrm{~mm}^{-1}$ or $1800 \mathrm{~g} \mathrm{~mm}^{-1}$ equipped with a thermoelectrically cooled CCD. The excitation laser with a wavelength of $532 \mathrm{~nm}$ from a diode laser was used for all Raman measurements. The laser power was set using WITec TruePower. The 2D-peak properties were extracted from the full-spectrum mapping results by fitting a single Lorentz after linear background subtraction.

\section{Temperature calibration}

Measurements were carried out under ambient conditions by placing the sample on a hotplate (Kammrath \& Weiss GmbH, LNT 250). The prepared membranes are clamped on a hot plate fixed on the piezo stage of the Raman microscope for mapping. To ensure thermalization of the membrane, a waiting time of $\sim 45 \mathrm{~min}$ is considered before each Raman map is acquired.

\section{$\mathrm{He}^{+}$-ion irradiation}

For the irradiation of freestanding graphene membranes, we used a He ion microscope (Orion, Zeiss) equipped with a pattern generator (Elphy MultiBeam, Raith) operated at $30 \mathrm{keV}$ using a probe current of $\sim 0.5 \mathrm{pA}$ at a chamber pressure of $\sim 7 \times 10^{-5} \mathrm{mbar}$. The ion dose was controlled by the exposure dwell time of each pixel ranging from 0.3 to $1.5 \mathrm{~ms}$.

\section{DATA AVAILABILITY}

The datasets analysed during the current study are available in the GitHub repository, https://github.com/MickaelPerrin74/ThermalconductivityMapping.

\section{CODE AVAILABILITY}

The code used during the current study is available in the GitHub repository, https:// github.com/MickaelPerrin74/ThermalconductivityMapping.

Received: 15 April 2021; Accepted: 30 November 2021; Published online: 17 January 2022

\section{REFERENCES}

1. Shi, L. et al. Evaluating broader impacts of nanoscale thermal transport research. Nanoscale Microscale Thermophys. Eng. 19, 127-165 (2015).

2. Song, $\mathrm{H}$. et al. Two-dimensional materials for thermal management applications. Joule 2, 442-463 (2018).

3. Corbino, O. M. Thermal oscillations in lamps of thin fibers with alternating current flowing through them and the resulting effect on the rectifier as a result of the presence of even-numbered harmonics. Phys. Z. 11, 413-417 (1910).

4. Corbino, O. M. Periodic resistance changes of fine metal threads which are brought together by alternating streams as well as deduction of their thermo characteristics at high temperatures. Phys. Z. 12, 292-295 (1911).

5. Seol, J. H. et al. Two-dimensional phonon transport in supported graphene. Science 328, 213-216 (2010).

6. Swinkels, M. Y. et al. Diameter dependence of the thermal conductivity of $\ln A s$ nanowires. Nanotechnology 26, 385401 (2015).

7. Yazji, S. et al. Assessing the thermoelectric properties of single InSb nanowires: The role of thermal contact resistance. Semicond. Sci. Technol. 31, 064001 (2016).

8. Deshpande, V. V., Hsieh, S., Bushmaker, A. W., Bockrath, M. \& Cronin, S. B. Spatially resolved temperature measurements of electrically heated carbon nanotubes. Phys. Rev. Lett. 102, 105501 (2009).

9. Soini, M. et al. Thermal conductivity of GaAs nanowires studied by micro-Raman spectroscopy combined with laser heating. Appl. Phys. Lett. 97, 263107 (2010).

10. Reparaz, J. S. et al. A novel contactless technique for thermal field mapping and thermal conductivity determination: two-laser Raman thermometry. Rev. Sci. Instrum. 85, 034901 (2014).
11. Neogi, S. et al. Tuning thermal transport in ultrathin silicon membranes by surface nanoscale engineering. ACS Nano 9, 3820-3828 (2015).

12. Shahil, K. M. \& Balandin, A. A. Thermal properties of graphene and multilayer graphene: Applications in thermal interface materials. Solid State Commun. 152, 1331-1340 (2012).

13. Balandin, A. A. Phononics of graphene and related materials. ACS Nano 14, 5170-5178 (2020).

14. Kasirga, T. S. Thermal Conductivity Measurements in Atomically Thin Materials and Devices. Springer Singapore, Singapore (2020).

15. Balandin, A. A. et al. Superior thermal conductivity of single-layer graphene. Nano Lett. 8, 902-907 (2008).

16. Ghosh, S. et al. Extremely high thermal conductivity of graphene: Prospects for thermal management applications in nanoelectronic circuits. Appl. Phys. Lett. 92, 151911 (2008).

17. Faugeras, $C$. et al. Thermal conductivity of graphene in corbino membrane geometry. ACS Nano 4, 1889-1892 (2010).

18. Lee, J.-U., Yoon, D., Kim, H., Lee, S. W. \& Cheong, H. Thermal conductivity of suspended pristine graphene measured by Raman spectroscopy. Phys. Rev. B 83, 081419(R) (2011).

19. Cai, W. et al. Thermal transport in suspended and supported monolayer graphene grown by chemical vapor deposition. Nano Lett. 10, 1645-1651 (2010).

20. Chen, S. et al. Raman measurements of thermal transport in suspended monolayer graphene of variable sizes in vacuum and gaseous environments. ACS Nano 5, 321-328 (2011).

21. Chen, S. et al. Thermal conductivity of isotopically modified graphene. Nat. Mater. 11, 203-207 (2012).

22. Lee, W. et al. In-plane thermal conductivity of polycrystalline chemical vapor deposition graphene with controlled grain sizes. Nano Lett. 17, 2361-2366 (2017).

23. Ma, T. et al. Tailoring the thermal and electrical transport properties of graphene films by grain size engineering. Nat. Commun. 8, 14486 (2017).

24. Chen, S. et al. Thermal conductivity measurements of suspended graphene with and without wrinkles by micro-Raman mapping. Nanotechnology 23, 365701 (2012).

25. Zhao, W. et al. Defect-engineered heat transport in graphene: a route to high efficient thermal rectification. Sci. Rep. 5, 11962 (2015).

26. Malekpour, H. et al. Thermal conductivity of graphene with defects induced by electron beam irradiation. Nanoscale 8, 14608-14616 (2016).

27. Ziabari, A. et al. Full-field thermal imaging of quasiballistic crosstalk reduction in nanoscale devices. Nat. Commun. 9, 255 (2018).

28. Majumdar, A. Scanning thermal microscopy. Annu. Rev. Mater. Sci. 29, 505-585 (1999).

29. Kim, K., Jeong, W., Lee, W. \& Reddy, P. Ultra-high vacuum scanning thermal microscopy for nanometer resolution quantitative thermometry. ACS Nano 6 , 4248-4257 (2012).

30. Menges, F. et al. Temperature mapping of operating nanoscale devices by scanning probe thermometry. Nat. Commun. 7, 10874 (2016).

31. Kinkhabwala, A. A., Staffaroni, M., Suzer, O., Burgos, S. \& Stipe, B. Nanoscale thermal mapping of HAMR heads using polymer imprint thermal mapping. IEEE Trans. Magn. 52, 1-4 (2016).

32. Mecklenburg, M. et al. Thermal measurement. nanoscale temperature mapping in operating microelectronic devices. Science 347, 629-632 (2015).

33. Calizo, I., Balandin, A. A., Bao, W., Miao, F. \& Lau, C. N. Temperature dependence of the Raman spectra of graphene and graphene multilayers. Nano Lett. 7, 2645-2649 (2007).

34. Dolleman, R. J. et al. Optomechanics for thermal characterization of suspended graphene. Phys. Rev. B 96, 475 (2017).

35. Abbe, E. K. Beiträge zur theorie des mikroskops und der mikroskopischen wahrnehmung. Arch. Mikrosk. Anat. 9, 413-468 (1873).

36. Pettes, M. T., Jo, I., Yao, Z. \& Shi, L. Influence of polymeric residue on the thermal conductivity of suspended bilayer graphene. Nano Lett. 11, 1195-1200 (2011).

37. Jo, I. et al. Reexamination of basal plane thermal conductivity of suspended graphene samples measured by electro-thermal micro-bridge methods. AIP Adv. 5, 053206 (2015).

38. $\mathrm{Xu}, \mathrm{X}$. et al. Length-dependent thermal conductivity in suspended single-layer graphene. Nat. Commun. 5, 3689 (2014).

39. Mercado, E., Anaya, J. \& Kuball, M. Impact of polymer residue level on the in-plane thermal conductivity of suspended large-area graphene sheets. ACS Appl. Mater. Interfaces 13, 17910-17919 (2021).

40. Buchheim, J., Wyss, R. M., Shorubalko, I. \& Park, H. G. Understanding the interaction between energetic ions and freestanding graphene towards practical $2 \mathrm{D}$ perforation. Nanoscale 8, 8345-8354 (2016).

41. Iberi, V. et al. Nanoforging single layer $\mathrm{MoSe}_{2}$ through defect engineering with focused helium ion beams. Sci. Rep. 6, 30481 (2016).

42. El Abbassi, M. et al. Benchmark and application of unsupervised classification approaches for univariate data. Commun. Phys. 4, 85 (2021). 
43. Eckmann, A. et al. Probing the nature of defects in graphene by Raman spectroscopy. Nano Lett. 12, 3925-3930 (2012).

44. Bae, M.-H. et al. Ballistic to diffusive crossover of heat flow in graphene ribbons Nat. Commun. 4, 1734 (2013).

45. El Sachat, A. et al. Crossover from ballistic to diffusive thermal transport in suspended graphene membranes. 2D Mater. 6, 025034 (2019).

46. Vakulov, D. et al. Ballistic phonons in ultrathin nanowires. Nano Lett. 20 2703-2709 (2020).

47. Fugallo, G. et al. Thermal conductivity of graphene and graphite: collective excitations and mean free paths. Nano Lett. 14, 6109-6114 (2014).

48. Cepellotti, A. et al. Phonon hydrodynamics in two-dimensional materials. Nat. Commun. 6, 6400 (2015).

49. Simoncelli, M., Marzari, N. \& Cepellotti, A. Generalization of Fourier's law into viscous heat equations. Phys. Rev. X 10, 66 (2020).

50. Luo, Z. et al. Anisotropic in-plane thermal conductivity observed in few-layer black phosphorus. Nat. Commun. 6, 8572 (2015).

51. Kang, J. S., Wu, H. \& Hu, Y. Thermal properties and phonon spectral characterization of synthetic boron phosphide for high thermal conductivity applications. Nano Lett. 17, 7507-7514 (2017).

52. Islam, A., van den Akker, A. \& Feng, P. X.-L. Anisotropic thermal conductivity of suspended black phosphorus probed by opto-thermomechanical resonance spectromicroscopy. Nano Lett. 18, 7683-7691 (2018).

53. Celebi, K. et al. Ultimate permeation across atomically thin porous graphene. Science 344, 289-292 (2014).

54. Thodkar, K. et al. Comparative study of single and multi domain CVD graphene using large-area Raman mapping and electrical transport characterization. Phys. Status Solidi RRL 10, 807-811 (2016).

55. Braun, O. et al. Optimized graphene electrodes for contacting graphene nanoribbons. Carbon 184, 331-339 (2021).

\section{ACKNOWLEDGEMENTS}

This work was supported by the EC H2020 FET Open project no. 767187 (QuIET). M.L.P. acknowledges funding by the EMPAPOSTDOCS-II program, which has received funding from the European Union's Horizon 2020 research and innovation program under the Marie Skłodowska-Curie Grant Agreement no. 754364. M.L.P. also acknowledges funding from the Swiss National Science Foundation under the Spark grant no. 196795. I.Z. and M.C. acknowledge funding from the Swiss National Science Foundation under the Sinergia grant no. 189924 (Hydronics). I.Z. acknowledges funding from the European Research Council (ERC) under the European Union's Horizon 2020 research and innovation program (Grant Agreement 756365). The author acknowledge support from the Multiphysics Hub @ Empa for the COMSOL Multiphyics calculations. We thank the Cleanroom Operations Team of the Binnig and Rohrer Nanotechnology Center (BRNC) for their help and support, and Roman M. Wyss for fruitful discussions and supply of $\mathrm{Si}_{3} \mathrm{~N}_{4}$ frames. We further thank Jan Overbeck, Maria El Abbassi, Marta De Luca and Milo Y. Swinkels for fruitful discussions.

\section{AUTHOR CONTRIBUTIONS}

O.B., I.S., M.C., and M.L.P. conceived and designed the experiments. K.T. developed the graphene growth recipe and transfer process. R.F. performed the graphene growth. O.B and I.S. prepared the SiN frame and performed the defect engineering using FIB. O.B. performed the Raman measurements. O.B., M.L.P., M.C., and I.Z. did the Raman spectroscopy analysis. P.B. developed the finite-element model to calculate the temperature distribution for a single laser spot position. M.L.P. extended the model to construct the temperature map upon illumination by the Raman laser and developed the procedure to fit the thermal conductivity. M.L.P. performed all finite-element calculations in the manuscript and supervised the study. O.B., M.L.P., and M.C. wrote the manuscript. All authors discussed the results and implications and commented on the manuscript.

\section{COMPETING INTERESTS}

The authors declare no competing interests.

\section{ADDITIONAL INFORMATION}

Supplementary information The online version contains supplementary material available at https://doi.org/10.1038/s41699-021-00277-2.

Correspondence and requests for materials should be addressed to Mickael L. Perrin.

Reprints and permission information is available at http://www.nature.com/ reprints

Publisher's note Springer Nature remains neutral with regard to jurisdictional claims in published maps and institutional affiliations.

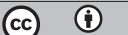

Open Access This article is licensed under a Creative Commons Attribution 4.0 International License, which permits use, sharing, adaptation, distribution and reproduction in any medium or format, as long as you give appropriate credit to the original author(s) and the source, provide a link to the Creative Commons license, and indicate if changes were made. The images or other third party material in this article are included in the article's Creative Commons license, unless indicated otherwise in a credit line to the material. If material is not included in the article's Creative Commons license and your intended use is not permitted by statutory regulation or exceeds the permitted use, you will need to obtain permission directly from the copyright holder. To view a copy of this license, visit http://creativecommons. org/licenses/by/4.0/.

(c) The Author(s) 2022 